\title{
RET Rearrangement as a Predictor of Unresponsiveness to Immunotherapy in Non-Small Cell Lung Cancer: Report of Two Cases with Review of the Literature
}

\author{
Sara Baglivo • Vienna Ludovini - Riccardo Moretti - Guido Bellezza • \\ Angelo Sidoni · Fausto Roila · Giulio Metro
}

Received: April 2, 2020 / Published online: May 20, 2020

(C) The Author(s) 2020

\section{ABSTRACT}

Patients with epidermal growth factor receptor and anaplastic lymphoma kinase positive nonsmall cell lung cancer (NSCLC) generally respond poorly to treatment with immune checkpoint inhibitors such as anti-programmed cell death-1 (PD-1) or anti-programmed cell death ligand-1 (PD-L1) given with or without

Digital Features To view digital features for this article go to https://doi.org/10.6084/m9.figshare.12162030.

\section{S. Baglivo · V. Ludovini}

Laboratory of Oncology, Medical Oncology, Santa

Maria della Misericordia Hospital, Azienda

Ospedaliera di Perugia, Perugia, Italy

\section{R. Moretti}

Department of Radiology, Santa Maria della Misericordia Hospital, Azienda Ospedaliera di Perugia, Perugia, Italy

G. Bellezza · A. Sidoni

Department of Experimental Medicine, Division of Pathology and Histology, University of Perugia

Medical School, Perugia, Italy

F. Roila · G. Metro ( $\square)$

Medical Oncology, Santa Maria della Misericordia Hospital, Azienda Ospedaliera di Perugia, Perugia, Italy

e-mail: giulio.metro@yahoo.com anti-cytotoxic T lymphocyte antigen-4 (CTLA4) drugs. However, the efficacy of immunotherapy in patients with oncogene-addicted NSCLC harboring minor drivers, such as fusions in the rearranged during transfection (RET) gene, is still unclear. Here we describe two patients with RET-positive advanced NSCLC with PD-L1 expression $\geq 50 \%$ who developed progressive disease during first-line treatment with the anti-PD-1 agent pembrolizumab. In particular, while patient 2 was immediately switched to treatment with a selective RET inhibitor within the setting of a clinical trial, patient 1 responded to cytotoxic chemotherapy delivered at the time of progression while on pembrolizumab. These cases of NSCLC are discussed in the context of current literature, which seems to support our observation that patients with RET-positive NSCLC are unlikely to benefit from immunotherapy. Therefore, we suggest that for RET-positive patients with PD$\mathrm{L} 1 \geq 50 \%$, consideration should be given to upfront treatment approaches other than single-agent immunotherapy, namely selective RET inhibitors (if available) or regimens including cytotoxic chemotherapy.

Keywords: Hyper-progressive disease; Immunotherapy; Non-small cell lung cancer; PD-L1 $\geq 50 \%$; Pembrolizumab; RET 


\section{Key Summary Points}

Rearranged during transfection (RET) gene rearrangements are found in approximately $2 \%$ of patients with nonsmall cell lung cancer (NSCLC). Importantly, the presence of these rearrangements predicts patient response to treatment with selective RET inhibitors.

The efficacy of immune checkpoint inhibitors in RET-positive NSCLC, however, has not yet been sufficiently established.

We describe two patients with RETpositive advanced NSCLC with antiprogrammed cell death-ligand 1 (PD-L1)

$\geq 50 \%$ who experienced progressive disease on first-line immunotherapy with pembrolizumab.

Our data, together with data reported in the literature, suggest that patients with RET-positive NSCLC are unlikely to benefit from immunotherapy.

Rather, selective RET inhibitors (if available) or cytotoxic chemotherapy (with or without immunotherapy) should be considered as the preferred treatment option in this context, regardless of the PD-L1 status of the tumor.

\section{INTRODUCTION}

In the last 15 years, advances in the molecular characterization of non-small cell lung cancer (NSCLC) have led to the identification of a number of genetic alterations that configure molecularly defined subgroups of oncogeneaddicted disease that is strictly dependent on the presence of a single actionable driver [1]. Epidermal growth factor receptor (EGFR) mutation and anaplastic lymphoma kinase (ALK) gene rearrangements were the first oncogenic drivers to be identified, and established targeted therapies of these rearrangements have been established, with excellent results. More recently, minor drivers, such as ROS protooncogene 1 receptor tyrosine kinase (ROS1) fusions, v-raf murine sarcoma viral oncogene homolog B1 (BRAF) mutations, human epidermal growth factor receptor 2 (HER2) mutations, hepatocyte growth factor receptor (MET) gene alterations, neurotrophin receptor kinase (NTRK) fusions, and rearranged during transfection (RET) fusions, have emerged as other important actionable alterations [1]. In particular, RET rearrangements have been recently added to the list of druggable target pathways, since selective RET inhibitors have shown impressive clinical activity in early clinical trials [2-4]. However, it is imperative that the oncologic community know whether therapies such as chemotherapy and/or immune checkpoint inhibition (ICI) with anti-programmed cell death-1 (PD-1) or anti-programmed cell death ligand-1 (PD-L1), with or without anti-cytotoxic $\mathrm{T}$ lymphocyte antigen-4 (CTLA-4) agents, are effective in treating oncogene-addicted NSCLC in order to select the best treatment strategy. While the unresponsiveness to immunotherapy of EGFR- and ALK-positive NSCLCs has been well described, the efficacy of ICI treatment in patients whose tumor harbors a minor oncogenic driver, such as a RET rearrangement, is still uncertain [5]. Here, we describe two patients with RET-positive advanced NSCLC with PD-L1 expression $\geq 50 \%$ who progressed on single-agent immunotherapy with the antiPD1 agent pembrolizumab administered as firstline treatment. We also discuss these cases in the context of the current literature.

Written informed consent was provided by both patients to published their respective case in an anonymous form.

\section{CASE 1}

A 59-year-old man, a never smoker, was diagnosed by trans-bronchial biopsy during bronchoscopy with a TTF1-positive lung adenocarcinoma of the right upper lobe. The tumor was classified as stage T4 with mediastinal infiltration, N2, and M1c for multiple 


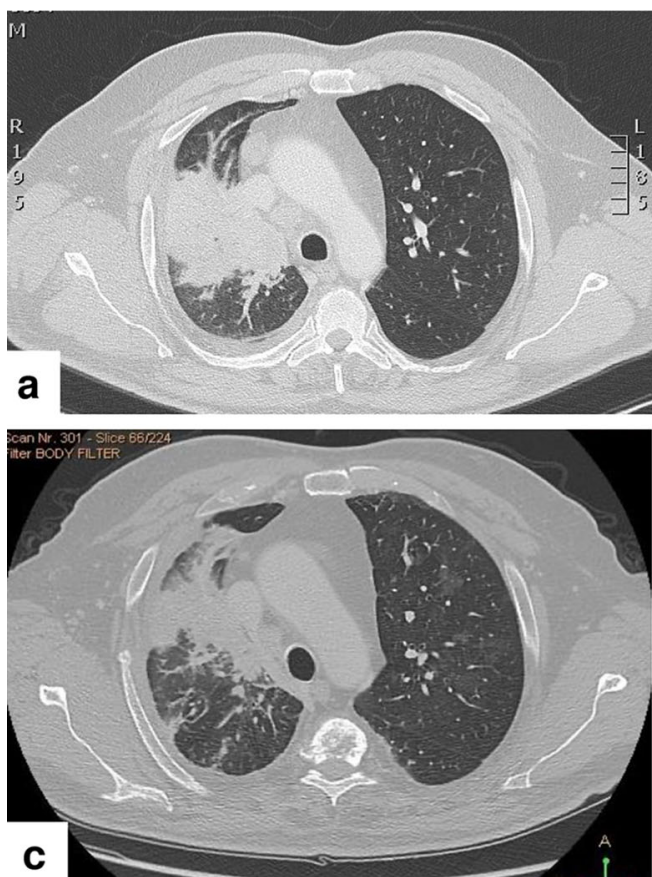

Fig. 1 a Computed tomography scan showing solid tissue localized at the lung hilum that infiltrates the right upper lobe bronchus with concomitant atelectasis of pulmonary parenchyma. b Progression of the solid tissue with an increase in atelectasis after one cycle of pembrolizumab. c Reduction in both the size of solid tissue and pulmonary

hepatic and bone lesions based on computed tomography (CT) scan of the thorax and abdomen (Fig. 1a). Magnetic resonance imaging (MRI) of the brain revealed an asymptomatic left-sided occipital lesion of $8 \mathrm{~mm}$ without surrounding edema. Immunohistochemistry analysis showed a PD-L1 tumor proportion score (TPS) of $60 \%$ (clone 22C3) (Fig. 2a), but no staining for ALK (clone D5F3) and ROS1 (clone D4D6). Next generation sequencing (NGS) (Ion Ampliseq Colon and Lung Research Panel v.2; Illumina, Inc., San Diego, CA, USA) showed that there was no EGFR mutation. Therefore, based on PD-L1 expression $\geq 50 \%$, the patient was advised to start treatment with pembrolizumab monotherapy at the flat dose of $200 \mathrm{mg}$ intravenously (i.v.) every 3 weeks. After only one cycle of therapy, however, he complained of worsening of pre-existing dyspnoea, with blood chemistry analysis showing mild alterations in the liver function tests. A new CT scan of the thorax and abdomen showed unequivocal

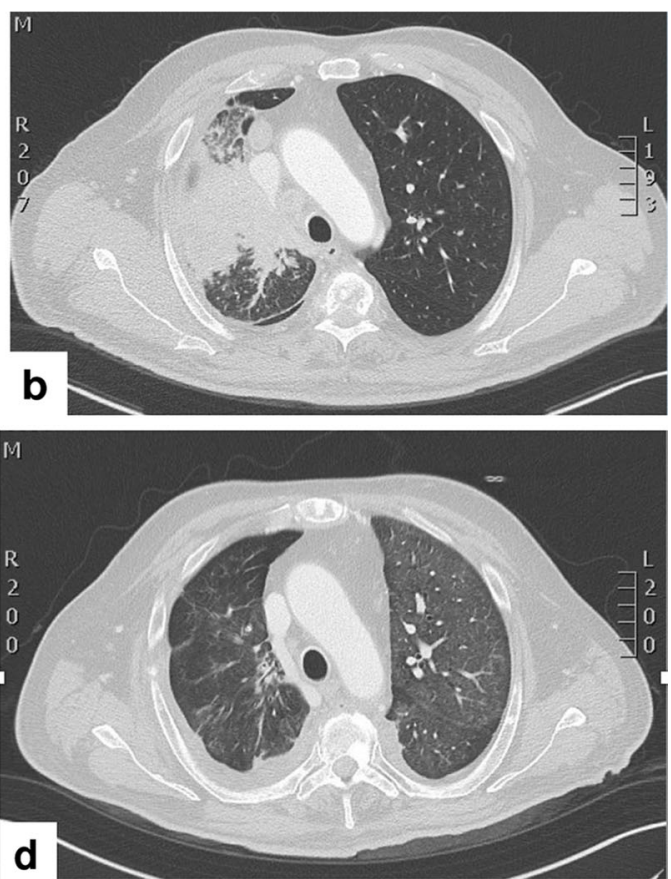

parenchyma after two cycles of chemotherapy. d Further reduction in the size of solid tissue with absence of atelectasis after 3 weeks of treatment with the RET (rearranged during transfection) inhibitor pralsetinib

progressive disease in both the lung (Fig. 1b) and liver. At that time, NGS for fusion transcripts was performed (Ion Ampliseq RNA Fusion Lung Cancer Research Panel), with the results being compatible with the presence of a RET rearrangement (KIF5B) (Fig. 2b). As the patient was experiencing symptomatic progression with possible hyper-progressive disease, treatment with pembrolizumab was stopped despite the patient having received only one cycle, and treatment was initiated with the cytotoxic agents carboplatin AUC $6+$ paclitaxel $90 \mathrm{mg} / \mathrm{m}^{2}$ i.v. on days 1,8 and 15 , with a cycle of chemotherapy every 3 weeks. After two cycles the patient reported an improvement in dyspnoea, while liver function tests showed progressive resolution; consistent with these results, a CT scan showed response to treatment (Fig. 1c). Based on his improved clinical condition, the patient was advised to start treatment with the selective RET-inhibitor pralsetinib on a once-daily dosing regimen of $400 \mathrm{mg}$ taken 

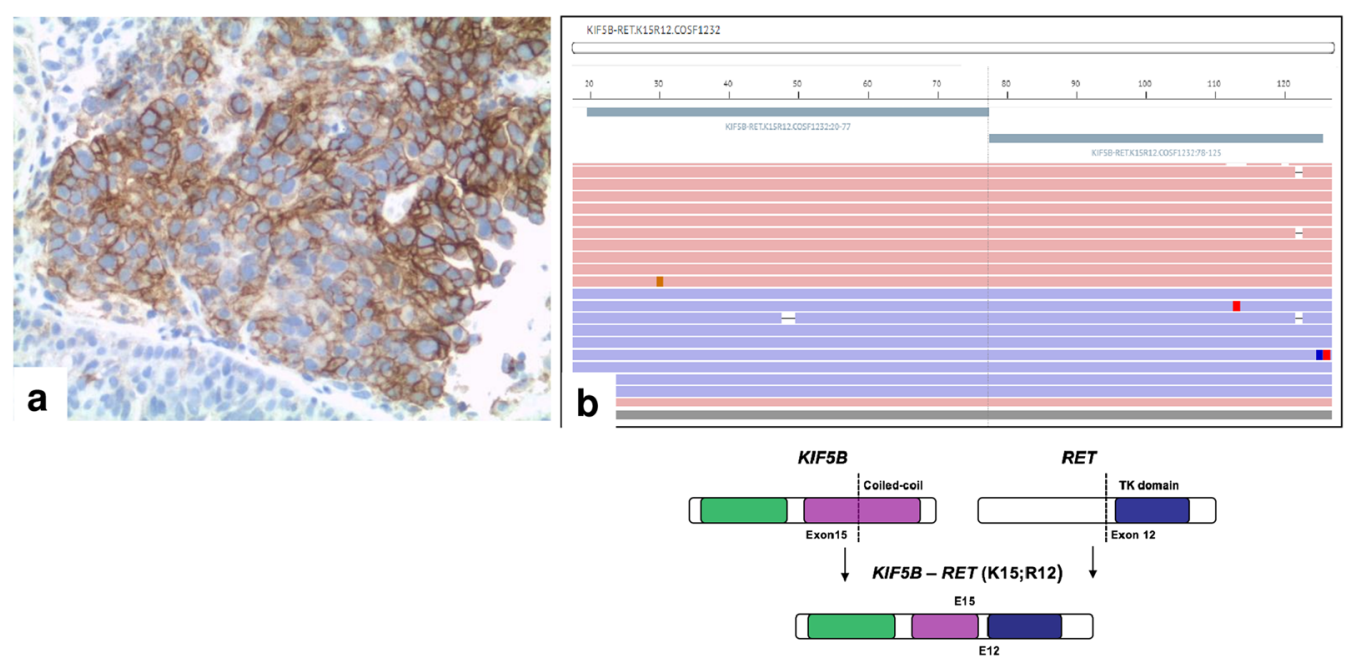

Fig. 2 a High expression (tumor proportion score: 60\%) of programmed cell death ligand-1 (PD-L1; clone 22C3) in neoplastic cells; for comparison, see negative staining in normal epithelium (lower left corner). b Next generation sequencing showing the presence of fusion transcript

orally within an expanded access program. A new CT scan of the thorax and abdomen and brain MRI performed only 3 weeks after the initiation of treatment with pralsetinib showed nearly complete resolution of the lung lesion (Fig. 1d), partial response in the liver, and reduction in the size of the brain lesion.

\section{CASE 2}

A 53-year-old woman, a never smoker, who had been complaining of a dry cough during the previous 2 months, underwent a CT scan of the thorax and abdomen that revealed the presence of a lung lesion of the left upper lobe with homolateral lymph-node enlargement at station 4L. A MRI of the brain revealed a small cortical lesion of $7 \mathrm{~mm}$ located at the left parasagittal site with mild edema, for which she was asymptomatic. Positron emission tomography/CT revealed no other extra-cranial lesions. An endobronchial ultrasound bronchoscopy with fine needle aspiration of the station $4 \mathrm{~L}$ lymph node was performed, and the results were compatible with TTF1-positive adenocarcinoma. Immunohistochemistry analysis revealed the following disease profile: PD-L1
KIF5B-RET (K15; R12)-COSF1232. The gene rearrangement involves the fusion of KIF5B exon 15 and RET exon 12

TPS score of 55\% (clone 22C3), ALK negative (clone D5F3), and ROS1 negative (clone D4D6). No EGFR mutation was detected by NGS (Ion Ampliseq Colon and Lung Research Panel v.2). Based on the high PD-L1 expression the patient was advised to start first-line treatment with pembrolizumab at the flat dose of $200 \mathrm{mg}$ i.v. every 3 weeks. After one cycle, the patient complained of worsening of the cough with onset of occasional hemoptysis, while a new CT scan showed unequivocal progressive disease at both the primary tumor and mediastinal lymph node. As in case 1 , rapid worsening of clinical conditions after only one cycle of pembrolizumab suggested the presence of hyperprogressive disease. At that time, a more comprehensive mutational analysis of fusion genes (Ion Ampliseq RNA Fusion Lung Cancer Research Panel) revealed the presence of a RET rearrangement (KIF5B). As a result, the patient was enrolled into a phase $1 / 2$ clinical trial evaluating the selective RET inhibitor pralsetinib (BLU-667) in RET-positive advanced malignancies ('ARROW' trial, ClinicalTrials.gov Identifier: NCT03037385). 


\section{DISCUSSION}

RET rearrangements are found in approximately $2 \%$ of patients with NSCLC [2]. If present, they confer a survival advantage to the tumor through constitutive activation of multiple downstream signaling pathways. RET rearrangements are more commonly found in young patients who have a never/light smoking history [2], as was the case for the two RETpositive patients described here. Importantly, knowing the baseline RET status of patients with newly diagnosed advanced NSCLC is of paramount importance as selective RET inhibitors with impressive clinical efficacy in treating RET-positive disease have recently become available. Pralsetinib and selpercatinib are two highly selective RET inhibitors that have achieved outstanding and durable response rates of $60-68 \%$ in patients with RET-positive NSCLC [3, 4]. However, the current availability of these drugs in the clinical setting is quite limited, and they are mostly accessible through participation in a clinical trial or through expanded access programs.

Against this background, it is important to pinpoint the sensitivity of RET-positive NSCLC tumors to treatments that are currently available, such as cytotoxic chemotherapy or ICI with anti-PD-(L) 1 as a single agent or in combination with anti-CTLA-4. With regard to cytotoxic treatments, RET-positive tumors appear to be particularly sensitive to pemetrexed-based chemotherapy, with responses observed in $45-63 \%$ of patients and median progression-free survival ranging from 9.0 to 19.0 months [6-8]. However, it is still unclear whether RET-positive NSCLC is sensitive to ICI treatment. We performed a literature search on this subject and identified three published reports describing the response of RET-positive NSCLC to ICI treatment administered either as monotherapy or as dual combination therapy (Table 1) [9-11]. Two of these reports describe very disappointing results for ICI treatment, with responses in only a very few patients. In line with these findings, both patients in the present report experienced hyper-progressive disease during first-line treatment with the antiPD1 agent pembrolizumab. When analyzed cumulatively with other studies, we found a disappointing response rate of $10.2 \%(4 / 39)$, a stable disease rate of $20.6 \%(10 / 39)$, and a progressive disease rate as high as $64.1 \%(25 / 39)$. Therefore, it is likely that RET-positive tumors, similar to other oncogene-addicted NSCLC

Table 1 Studies reporting on the efficacy of immunotherapy in RET (rearranged during transfection)-positive patients

\begin{tabular}{|c|c|c|c|c|c|c|c|}
\hline $\begin{array}{l}\text { First author } \\
\text { and year }\end{array}$ & $\begin{array}{l}\text { Immune } \\
\text { checkpoint } \\
\text { inhibition }\end{array}$ & $\begin{array}{l}\text { No. of } \\
\text { patients }\end{array}$ & $\begin{array}{l}\text { Partial } \\
\text { response }\end{array}$ & Stable disease & $\begin{array}{l}\text { Progressive } \\
\text { disease }\end{array}$ & $\begin{array}{l}\text { Median } \\
\text { progression-free } \\
\text { survival }\end{array}$ & $\begin{array}{l}\text { Median } \\
\text { overall } \\
\text { survival }\end{array}$ \\
\hline $\begin{array}{l}\text { Offin et al. } \\
(2019) \text { [9] }\end{array}$ & $\begin{array}{l}\text { Anti-PD- } \\
\text { (L) } 1 \pm \text { anti- } \\
\text { CTLA- } 4\end{array}$ & 13 & 0 & 5 & 8 & $3.4^{\mathrm{b}}$ & NR \\
\hline $\begin{array}{l}\text { Mazieres et al. } \\
(2019)[10]\end{array}$ & Anti-PD-(L)1 & 16 & 1 & 3 & 12 & 2.1 & 21.3 \\
\hline $\begin{array}{l}\text { Guisier et al. } \\
(2020)[11]\end{array}$ & Anti-PD-1 & 8 & 3 & 2 & 3 & 7.6 & NR \\
\hline $\begin{array}{l}\text { Present } \\
\text { report }\end{array}$ & Anti-PD-1 & 2 & 0 & 0 & 2 & - & - \\
\hline
\end{tabular}

CTLA-4 Cytotoxic T lymphocyte antigen-4, $N R$ not reported, $P D-1$ programmed cell death-1, $P D-L 1$ programmed cell death ligand-1

${ }^{\text {a }}$ Evaluable for response

b On 16 patients 
subtypes, belong to a poorly immunogenic subgroup in which a single mutation is assumed to act as driver of the respective cancer cells in the context of a poorly immunogenic tumor microenviroment [12]. In line with this hypothesis, RET-positive NSCLC has been associated with low levels of tumor mutational burden (TMB), which is considered a biomarker of sensitivity to immunotherapy [9]. In fact, by comparing RET-positive NSCLC subtypes with wild-type RET cancerous tumors, Offin et al. found a significantly lower median TMB for RET-positive cases. However, it is unknown if other molecular features might have contributed to the hyper-progression on pembrolizumab of our two patients. Recent data suggest that simultaneous PD-1 and PD-L1 expression in tumor cells could induce tumor suppression, which is antagonized by the use of anti-PD-(L)1 agents [13, 14]. Therefore, in the absence of an adaptive immune system, which could be the case of patients with RET-positive NSCLC lesions, anti-PD-(L) 1 agents may promote resistance to treatment.

Having said this, the question of whether PD-L1 status retains significance in determining sensitivity to ICI treatment in RET-positive NSCLC patients is relevant, as two competing standards exist in this setting, either pembrolizumab alone or pembrolizumab in combination with platinum-based chemotherapy. Importantly, PD-L1 does not appear to be expressed at high levels in RET-positive NSCLC; in the study by Offin et al., of 26 cases of RETpositive NSCLCs, only 19\% (5/26) had PD-L1 expression $\geq 50 \%$ [9]. Nevertheless, the question of will PD-L1 status impact the choice of first-line treatment in RET-positive NSCLC lesions remains. With regard to this, the two RET-positive cases presented herein are interesting because, despite the presence of PD-L1 levels $\geq 50 \%$, both were both unresponsive to treatment with the single-agent pembrolizumab. Similarly, progressive disease as best response was reported by Offin et al. in the only patient with PD-L1 expression $\geq 50 \%$ treated with ICI [9].

In summary, we conclude that knowing the RET status at baseline in newly diagnosed advanced NSCLC patients is crucial, especially in those individuals with PD-L1 $\geq 50 \%$. In the latter case, in countries with limited access to a selective RET-inhibitor, consideration should be given to the administration of chemotherapy with or without pembrolizumab rather than pembrolizumab alone, as these tumors are very unlikely to respond to ICI treatment. In fact, our cases suggest that PD-L1 status seems to have little predictive value in patients with RETpositive NSCLC as hyper-progressive disease was noted with pembrolizumab as the single agent despite the presence of PD-L1 TPS $\geq 50 \%$. In addition, knowing the poor responsiveness of RET-positive NSCLC to ICI treatment regardless of PD-L1 status can also help guide drug sequencing for patients who require salvage treatment at the time of progression on RETinhibitors. In fact, RET-positive patients who have not been treated with immunotherapy in the first-line setting are unlikely to benefit from ICI treatment in later lines.

\section{ACKNOWLEDGEMENTS}

We thank the patients for giving their consent to publish their cases.

Funding. No funding or sponsorship was received for this study or publication of this article.

Authorship. All named authors meet the International Committee of Medical Journal Editors (ICMJE) criteria for authorship for this article and take the responsibility for the integrity of the work as a whole, and have given their approval for this version to be published.

Authorship Contributions. Fausto Roila and Giulio Metro were responsible for the conception of the work, data collection, and manuscript writing. Sara Baglivo, Vienna Ludovini, Guido Bellezza, Angelo Sidoni, provided molecular data of the patients. Riccardo Moretti provided radiographic material. All authors read and gave their approval for the manuscript to be published in its final version. 
Disclosures. Sara Baglivo, Vienna Ludovini, Riccardo Moretti, Guido Bellezza, Angelo Sidoni, Fausto Roila, Giulio Metro have nothing to disclose.

Compliance with Ethics Guidelines. Written informed consent was provided from both patients for publishing their cases in an anonymous form.

Data Availability. All data generated or analyzed during this study are included in this published article.

Open Access. This article is licensed under a Creative Commons Attribution-NonCommercial 4.0 International License, which permits any non-commercial use, sharing, adaptation, distribution and reproduction in any medium or format, as long as you give appropriate credit to the original author(s) and the source, provide a link to the Creative Commons licence, and indicate if changes were made. The images or other third party material in this article are included in the article's Creative Commons licence, unless indicated otherwise in a credit line to the material. If material is not included in the article's Creative Commons licence and your intended use is not permitted by statutory regulation or exceeds the permitted use, you will need to obtain permission directly from the copyright holder. To view a copy of this licence, visit http://creativecommons.org/licenses/by$\mathrm{nc} / 4.0 /$.

\section{REFERENCES}

1. Dong J, Li B, Lin D, Zhou Q, Huang D. Advances in targeted therapy and immunotherapy for nonsmall cell lung cancer based on accurate molecular typing. Front Pharmacol. 2019;10:230.

2. Subbiah V, Yang D, Velcheti V, Drilon A, MericBernstam F. State-of-the-art strategies for targeting RET-dependent cancers. J Clin Oncol. 2020;38: 1209-21.

3. Gainor JF, Lee DH, Curigliano G, et al. Clinical activity and tolerability of BLU-667, a highly potent and selective RET inhibitor in patients (pts) with advanced RET-fusion+ non-small cell lung cancer (NSCLC). J Clin Oncol. 2019;37:abstract 9008.

4. Drilon A, Oxnard G, Wirth L, et al. Registrational results of LIBRETTO-001: a phase $1 / 2$ trial of LOXO292 in patients with RET fusion-positive lung cancers. J Thorac Oncol. 2019;14:abstract S6-S7.

5. Metro G, Di Maio M. Cons: should immunotherapy be incorporated in the treatment of oncogene-driven lung cancer? Transl Lung Cancer Res. 2018;7(Suppl 3):S294-S296296.

6. Drilon A, Bergagnini I, Delasos L, et al. Clinical outcomes with pemetrexed-based systemic therapies in RET-rearranged lung cancers. Ann Oncol. 2016;27:1286-91.

7. Lee J, Ku BM, Shim JH, et al. Characteristics and outcomes of RET-rearranged Korean non-small cell lung cancer patients in real-world practice. Jpn J Clin Oncol. 2020;50:594-601.

8. Shen T, Pu X, Wang L, et al. Association between RET fusions and the efficacy of pemetrexed-based chemotherapy for advanced NSCLC patients in China: a multicentre retrospective study. Clin Lung Cancer. 2020. https://doi.org/10.1016/j.cllc.2020. 02.006 .

9. Offin M, Guo R, Wu SL, et al. Immunophenotype and response to immunotherapy of RET-rearranged lung cancers. JCO Precis Oncol. 2019;3. https://doi. org/10.1200/PO.18.00386.

10. Mazieres J, Drilon A, Lusque A, et al. Immune checkpoint inhibitors for patients with advanced lung cancer and oncogenic driver alterations: results from the IMMUNOTARGET registry. Ann Oncol. 2019;30:1321-8.

11. Guisier F, Dubos-Arvis C, Viñas F, et al. Efficacy and safety of anti-PD-1 immunotherapy in patients with advanced non small cell lung cancer with BRAF, HER2 or MET mutation or RET-translocation. GFPC 01-2018. J Thorac Oncol. 2020;15:628-36.

12. Mhanna L, Guibert N, Milia J, Mazieres J. When to consider immune checkpoint inhibitors in oncogene-driven non-small cell lung cancer? Curr Treat Options Oncol. 2019;20:60.

13. Du S, Du S, McCall N, Park K, et al. Blockade of tumor-expressed PD-1 promotes lung cancer growth. Oncoimmunology. 2018;7:e1408747.

14. Wang $X$, Yang $X$, Zhang $C$, et al. Tumor cell intrinsic PD-1 receptor is a tumor suppressor and mediates resistance to PD-1 blockade therapy. Proc Natl Acad Sci USA. 2020;117:6640-50. 